\title{
Thermal and Photoinduced Control of Relative Populations of 4-Methoxybenzaldehyde ( $p$-Anisaldehyde) Conformers
}

\author{
Nihal Kuş, ${ }^{\dagger, \ddagger}$ Archna Sharma ${ }^{\dagger}$ Igor Reva, ${ }^{\dagger}$ Leszek Lapinski, ${ }^{\S}$ and Rui Fausto ${ }^{*, \dagger}$ \\ Department of Chemistry, University of Coimbra, 3004-535 Coimbra, Portugal, Department of Physics, \\ Anadolu University, 26470 Eskişehir, Turkey, and Institute of Physics, Polish Academy of Sciences, \\ Warsaw, Poland
}

Received: March 9, 2010; Revised Manuscript Received: June 2, 2010

\begin{abstract}
Two almost isoenergetic conformers of 4-methoxybenzaldehyde ( $p$-anisaldehyde), O-trans and O-cis, are nearly equally populated in gas phase at room temperature. The existence of these two conformers of similar energy makes $p$-anisaldehyde an attractive molecule for conformational investigations, in which the relative populations of the two forms might be subjected to optical control. In the present study, monomers of the compound were trapped from the room-temperature gas phase into cryogenic argon and xenon matrices. The initial relative amount of the two conformers present in the freshly deposited matrices is shifted slightly in favor of the O-trans conformer. The ratio of the two forms could be reversibly varied by irradiating the sample with UV light in different wavelength ranges or by using the temperature variation. Increasing the temperature of the xenon matrix up to ca. $57 \mathrm{~K}$ led to conversion of the less stable O-cis form into the O-trans conformer, shifting the O-cis/O-trans ratio to ca. 1:7. A series of UV irradiations with different long-pass cutoff filters was carried out. UV excitation induced transformation of O-cis and O-trans conformers into each other. These transformations were leading to the UV-wavelength-specific photostationary equilibria characterized by the O-cis/O-trans ratios of about 1:2.2, 1:1.4, 1:1.1, and 1:0.89 for $\lambda>328,295$, 288, and $234 \mathrm{~nm}$ cutoff filters, respectively. The isomerization processes were probed by infrared spectroscopy and supported by quantum chemical calculations. The absorption bands observed in the infrared spectra of $p$-anisaldehyde isolated in argon and xenon matrices were assigned to the theoretically predicted normal modes.
\end{abstract}

\section{Introduction}

4-Methoxybenzaldehyde (known also as p-anisaldehyde) is an aromatic organic compound that has vast industrial applications. It is used as an additive for metal plate brighteners and perfumes, and as a source material for medicines. $p$-Anisaldehyde is also used as an active ingredient in antifungal fragrance compositions as well as in the synthesis of nematic liquid crystals. Its binary mixtures are employed in high-performance thin layer chromatography, for example, in the process of separation of biological compounds. ${ }^{1-4}$

Chemically, $p$-anisaldehyde is produced through liquid-phase oxidation of $p$-methoxy-toluene. ${ }^{5} p$-Anisaldehyde has been reported to dimerize readily in solution due to hydrogen bond formation, ${ }^{6-8}$ and it is suggested that a charge transfer from the methoxy group to the aldehyde group is responsible for this type of aggregation. ${ }^{3}$

There are also a few reports related to $p$-anisaldehyde monomer. ${ }^{9-11}$ Microwave spectroscopy $y^{9,10}$ allowed the conclusion that the compound exists in gas phase as a mixture of two almost isoenergetic planar conformers, O-cis and O-trans (Figure 1). Their relative populations might be subjected to optical control. This possibility opens the gate for exciting conformational studies on $p$-anisaldehyde. The optical control of conformational compositions is of fundamental importance, for example, to the study of conformation-dependent photo-

\footnotetext{
* To whom correspondence should be addressed. E-mail: rfausto@ci.uc.pt.

$\uparrow$ University of Coimbra.

* Anadolu University.

$\S$ Polish Academy of Sciences.
}

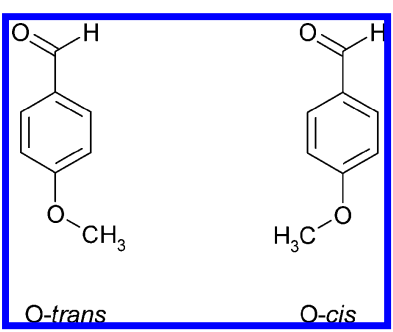

Figure 1. Conformers of $p$-anisaldehyde.

chemical reactions ${ }^{12-15}$ and may receive potential application in the building up of molecular devices (e.g., in the design of light-controlled molecular switches).

In the present study, we show that the relative amount of the two conformers of $p$-anisaldehyde in cryogenic matrices can be efficiently varied in both directions by irradiating the sample with UV light of different wavelengths. We also report a detailed analysis of the infrared spectra of $p$-anisaldehyde isolated in $\mathrm{Ar}$ and Xe cryogenic matrices.

\section{Experimental and Computational Methods}

A commercial sample of $p$-anisaldehyde (Aldrich, 99.5\%) was placed in a glass tube connected through a needle valve to the vacuum chamber of the cryostat. Before deposition of lowtemperature matrices, the sample was additionally purified by pumping in order to remove any volatile impurities. Roomtemperature vapor of $p$-anisaldehyde, passing through the needle valve and a nozzle connected to it, was deposited together with a large excess of argon (N60, Air Liquide) or xenon (N48, Air 
Liquide) onto an optical CsI substrate cooled to $10 \mathrm{~K}$ (Ar) or $30 \mathrm{~K}(\mathrm{Xe})$ by an APD Cryogenics DE-202A closed-cycle refrigerator. The infrared spectra of matrices were collected with $0.5 \mathrm{~cm}^{-1}$ resolution, using a Mattson (Infinity 60AR series) FTIR or a Thermo Nicolet Nexus 670 FTIR spectrometer, equipped with a DTGS detector and a KBr beam splitter.

Photoinduced transformations of matrix-isolated $p$-anisaldehyde were studied using two different sources of UV radiation. In the first approach, broadband UV light emitted by a high pressure $\mathrm{Xe} / \mathrm{Hg}$ lamp fitted with different long-pass cutoff filters (transmitting light with $\lambda>200,234,288,295,328 \mathrm{~nm}$ ) and 8 $\mathrm{cm}$ water filter was used. In the second approach, a narrowband $\left(0.2 \mathrm{~cm}^{-1}\right.$ spectral width) frequency-doubled UV beam $(\lambda=$ $280 \mathrm{~nm}$ ) provided by a Quanta-Ray MOPO-SL optical parametric oscillator (pumped with a pulsed Nd:YAG laser) was applied. The repetition rate, pulse energy, and duration were $10 \mathrm{~Hz}, 5 \mathrm{~mJ}$, and $10 \mathrm{~ns}$, respectively.

Geometry optimizations and calculations of infrared spectra were carried out at the DFT(B3LYP)/6-311++G(d,p) level ${ }^{16-18}$ using the Gaussian 03 program package. ${ }^{19}$ One- or twodimensional DFT scans of the potential energy surface (PES) of $p$-anisaldehyde molecule were performed by stepwise incrementing of dihedral angles determining rotations of the methoxy and/or aldehyde groups and optimizing (by energy minimization) the values of all other parameters describing the geometry of the molecule. The nature of the optimized stationary points was characterized by the analysis of the corresponding Hessian matrices. Additionally, for the electronic ground state of $p$-anisaldehyde, geometries of the minima and transition states were optimized using the MP2/6-311++G(d,p) method. ${ }^{20}$ At this theory level, calculation of the analytic MP2 second derivatives for $p$-anisaldehyde is not possible, due to the limitations of the 32-bit version of Gaussian. Therefore the MP2 frequencies were computed numerically. Energies of the $S_{\mathrm{i}}(i$ $=1,2,3$ ) low-energy excited singlet states were calculated using the time-dependent density functional theory (TD-DFT) ${ }^{21,22}$ at the (B3LYP)/6-311++G(d,p) level.

The theoretical normal modes were analyzed by carrying out the potential energy distribution (PED) calculations. Transformations of the force constants with respect to the Cartesian coordinates to the force constants with respect to the molecule fixed internal coordinates allowed the PED analysis to be carried out as described by Schachtschneider and Mortimer. ${ }^{23}$ The internal symmetry coordinates used in this analysis were defined as recommended by Pulay et al. ${ }^{24}$ and are listed in Table S1 of the Supporting Information. The elements of the computed PED matrices greater than $10 \%$ are presented in Tables S2 and S3 of the Supporting Information.

\section{Results and Discussion}

Theoretical Study of the Conformational Isomerism in p-Anisaldehyde. The DFT(B3LYP)/6-311++G(d,p) calculated ground state potential energy surface of $p$-anisaldehyde exhibits two different minima (Figure 2), corresponding to the O-trans and $\mathrm{O}$-cis conformers of the compound. The two conformers (shown in Figure 1) differ by the orientation of the methyl fragment (in the methoxy group) with respect to the oxygen atom of the aldehyde group. ${ }^{25}$ In agreement with the previously reported microwave studies, ${ }^{9,10}$ our calculations predict that the two conformers (both of $C_{\mathrm{s}}$ symmetry) are almost isoenergetic. The O-trans form is computed to be more stable than the O-cis form by only 0.57 (MP2) or 0.17 (DFT) $\mathrm{kJ} \mathrm{mol}^{-1}$ ( $E_{\mathrm{ZPE}}$, see Table S4 of the Supporting Information). This result could be intuitively expected, because the aldehyde and the methoxy groups are placed at remote positions of the molecule.

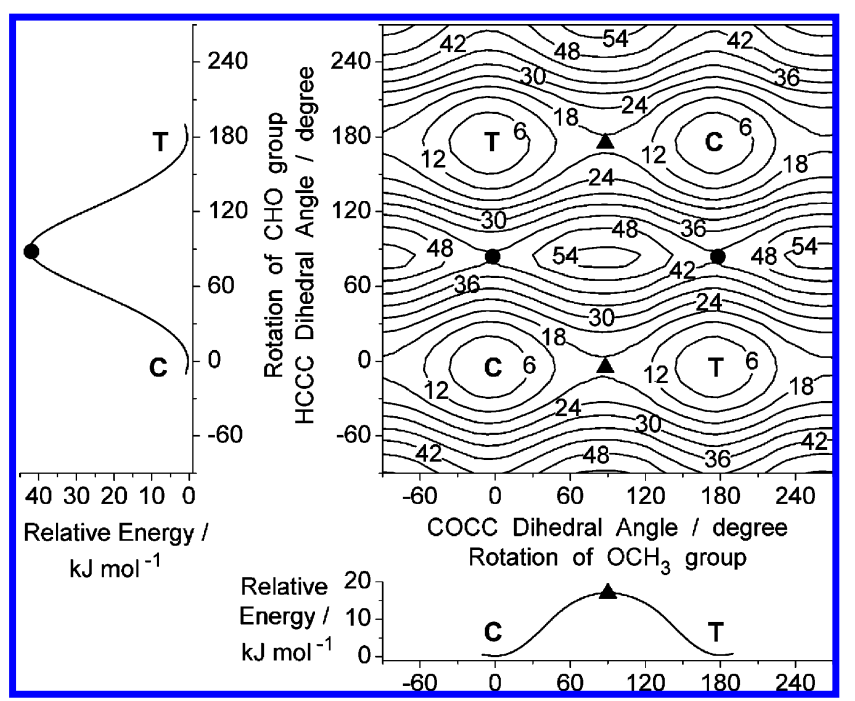

Figure 2. Two-dimensional potential energy surface of $p$-anisaldehyde. Energy was calculated at the DFT(B3LYP)/6-311++G(d,p) level as a function of internal rotation of the methoxy group (determined by the $\mathrm{C}-\mathrm{O}-\mathrm{C}-\mathrm{C}$ dihedral angle) and of internal rotation of the aldehyde group (determined by the $\mathrm{H}-\mathrm{C}-\mathrm{C}-\mathrm{C}$ dihedral angle). The driving coordinates (dihedral angles) were changed incrementally and all the remaining internal coordinates were fully optimized at each of the steps. Isoenergy levels are shown with a step of $6 \mathrm{~kJ} \mathrm{~mol}^{-1}$. Left and bottom projections: the corresponding one-dimensional potential energy scans (note that the linear energy scales are identical). $\mathbf{C}$ and $\mathbf{T}$ designate positions of O-cis and O-trans conformers, respectively. Circles and triangles designate positions of the transition states.

The barriers for O-cis $\leftrightarrow$ O-trans interconversion via rotation of the methoxy or aldehyde groups, calculated at the DFT level, amount to 17 and $43 \mathrm{~kJ} \mathrm{~mol}^{-1}$, respectively (see projections in Figure 2). However, it was reported that "molecules comprising a benzene ring with a $\pi$-conjugated substituent form a special problem with respect to calculation of the rotational barrier around the $\mathrm{C}\left(\mathrm{sp}^{2}\right)-\mathrm{C}(\operatorname{aryl})$ bond" and overestimate this barrier with current DFT methods. ${ }^{26,27}$ Therefore, we also computed at the MP2 level the barriers for internal rotation for anisaldehyde and model compounds, containing an aromatic ring connected to either aldehyde or methoxy fragment. These MP2 calculations produce values of all the barriers that are systematically lower than the DFT ones (see Table 1). However, at both MP2 and DFT levels, the calculated barriers for internal rotation of the methoxy group (ca. $10 \mathrm{~kJ} \mathrm{~mol}^{-1}$ ) are substantially (ca. 3 times) lower than the barriers for internal rotation of the aldehyde group (see Table 1).

The calculated value of the rotational barrier around the phenyl-methoxy linkage is in agreement with the experimental results of microwave studies of Lister $^{28}$ (no less than $4.6 \mathrm{~kJ}$ $\mathrm{mol}^{-1}$ in anisoles) and the electron diffraction study of Brunvoll et al. ${ }^{11}$ (no higher than $12 \mathrm{~kJ} \mathrm{~mol}^{-1}$ in $p$-anisaldehyde). These experimental values agree with calculated values for the barrier of rotation of the methoxy group in anisole, which is about 10 $\mathrm{kJ} \mathrm{mol}^{-1} \cdot 29,30$

On the other hand, the substantial height of the barrier for rotation of the aldehyde group may be attributed to conjugation between the $\pi$-electron systems of the carbonyl moiety and of the benzene ring. The presently accepted theoretical value for the aldehyde group rotational barrier in benzaldehyde ${ }^{31}$ is ca. $32 \mathrm{~kJ} \mathrm{~mol}^{-1}$ and is also in agreement with our MP2 calculations (see Table 1).

During deposition of low-temperature matrices, barriers to internal rotation play a very important role. In the case of very low barriers, the conformers with higher energies may relax to 
TABLE 1: Energies ( $\mathrm{kJ} \mathrm{mol}^{-1}$ ) of the Barriers for Conformational Isomerization, in the Electronic Ground State, Calculated Using the DFT(B3LYP)/6-311 ++ G(d,p) and MP2/6-311 $++G(d, p)$ Levels of Theory for $p$-Anisaldehyde and Model Compounds ${ }^{a}$

\begin{tabular}{|c|c|c|c|c|c|}
\hline No. ${ }^{b}$ & Structure $^{c}$ & Level & $E_{e l}^{d}$ & $E_{Z P E}^{e}$ & $E_{G}{ }^{f}$ \\
\hline \multirow{2}{*}{1} & & DFT & 43.1 & 40.9 & 41.9 \\
\hline & & MP2 & 33.9 & 32.5 & 34.0 \\
\hline \multirow{2}{*}{2} & & DFT & 36.7 & 34.3 & 35.5 \\
\hline & & MP2 & 30.3 & 27.0 & 28.5 \\
\hline \multirow{2}{*}{3} & & DFT & 52.0 & 49.6 & 50.5 \\
\hline & & MP2 & 42.5 & 42.9 & 45.2 \\
\hline \multirow{2}{*}{4} & $\mathrm{H}$ & DFT & 17.1 & 15.3 & 16.9 \\
\hline & $0^{\prime \prime}$ & MP2 & 11.4 & 9.1 & 10.9 \\
\hline \multirow{2}{*}{5} & & DFT & 12.3 & 10.8 & 12.5 \\
\hline & & MP2 & 8.2 & 9.6 & 13.9 \\
\hline
\end{tabular}

Information (Table S4). ${ }^{b}$ 1: $p$-anisaldehyde, rotation of the aldehyde group from O-cis to O-trans conformer (TS1); 2: benzaldehyde, rotation of the aldehyde group; 3: pyrrole-2-carboxaldehyde (P2C); rotation of the aldehyde group from trans to cis conformer; 4: $p$-anisaldehyde, rotation of the methoxy group from $\mathrm{O}$-cis to O-trans conformer (TS2); 5: anisole, rotation of the methoxy group. ${ }^{c}$ Arrows indicate the direction of rotation that occurs from the higher-energy to the lower-energy conformer (if applicable). ${ }^{d}$ Electronic energy. ${ }^{e}$ Zero-point vibrational energy corrected. ${ }^{f}$ Gibbs free energy at $298 \mathrm{~K}$.

lower-energy forms. ${ }^{32,33}$ This phenomenon, called conformational cooling, can make it impossible to trap higher-energy conformers in low-temperature solid noble gas environment. However, if the barriers are high enough, conformational cooling should be precluded and lower- as well as higher-energy forms should be trapped upon deposition of the matrices. ${ }^{34}$ The heights of the barriers theoretically predicted for $p$-anisaldehyde suggest that both O-trans and O-cis forms of the compound should be frozen in the matrices.

Experimental Study of the Conformational Isomerism in p-Anisaldehyde. The relative populations of O-trans and O-cis conformers of $p$-anisaldehyde in gas phase [at $298.15 \mathrm{~K}$ (25 ${ }^{\circ} \mathrm{C}$ ), which corresponds to the temperature of the vapor of the compound immediately before matrix deposition] can be theoretically estimated from their relative Gibbs energies and the Boltzmann distribution. The MP2 calculations predict the free Gibbs energy difference of $0.57 \mathrm{~kJ} \mathrm{~mol}^{-1}$ in favor of O-trans (Table S4), the population ratio $\mathrm{O}$-cis/O-trans, at room temperature, can be expected to be $45 \%: 55 \%$ (or 1:1.2).

The FTIR spectra recorded immediately after deposition of $p$-anisaldehyde in argon $(10 \mathrm{~K})$ and xenon $(30 \mathrm{~K})$ matrices are presented in Figure 3, where they can be compared with the theoretical spectra calculated for the two conformers of the compound. This figure also shows the simulated IR spectrum of $p$-anisaldehyde obtained by adding the theoretical spectra of the two conformers with weights equal to their theoretically

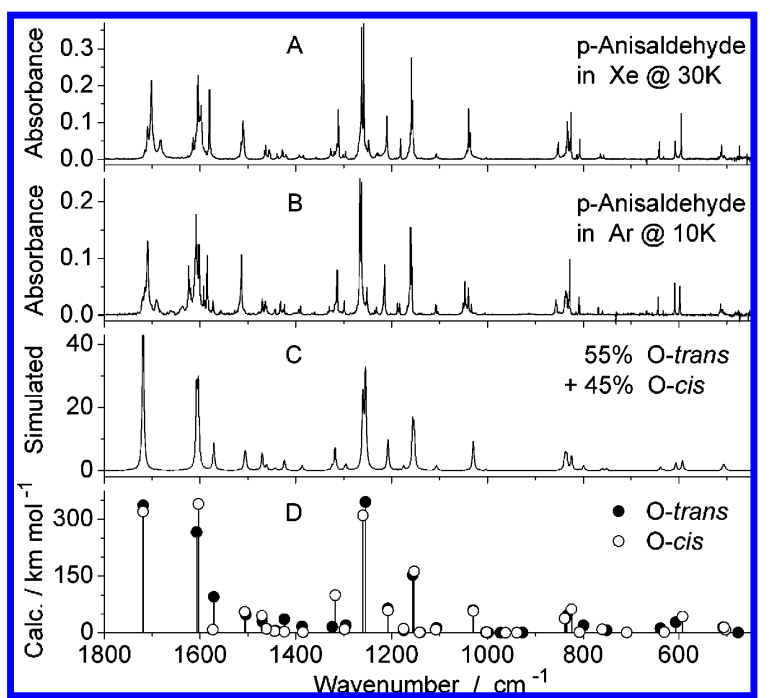

Figure 3. A and B: Experimental FTIR spectra of $p$-anisaldehyde isolated in xenon $(30 \mathrm{~K})$ and $\operatorname{argon}(10 \mathrm{~K})$ matrices. D: Theoretical spectra of O-trans and O-cis conformers calculated at the DFT(B3LYP)/ $6-311++\mathrm{G}(\mathrm{d}, \mathrm{p})$ level. The calculated wavenumbers were scaled by a factor of 0.978. C: Theoretical superposition spectrum of the mixture of two conformers simulated with Lorentzian functions (fwhm $=2$ $\mathrm{cm}^{-1}$ ) centered at the calculated (scaled) frequencies. The calculated intensities were weighted by the equilibrium Boltzmann populations of the two conformers at $298.15 \mathrm{~K}$, as estimated from the MP2/6$311++\mathrm{G}(\mathrm{d}, \mathrm{p})$ calculations.

predicted room temperature equilibrium populations. As it can be seen in the figure, the simulated spectrum reproduces well the experimental data. The proposed band assignments are collected in Table 2. The full list of calculated frequencies and IR intensities as well as the results of the normal coordinate analysis (carried out for both conformers of $p$-anisaldehyde) are provided in the Supporting Information (Tables S1-S3).

It is worth mentioning that, over the entire fingerprint region of the theoretically calculated spectra, the pairs of IR bands due to O-trans and O-cis conformers either overlap or appear as doublets at close frequencies. These theoretical predictions are in good agreement with the experimental observations. As shown below in detail, the assignment of components of doublet bands to a particular conformer was facilitated by experiments where the population ratio of O-trans and O-cis conformers was altered either by changing the temperature of the matrix or by irradiating the matrix with UV light.

O-cis $\rightarrow$ O-trans Conformational Conversion upon Matrix Annealing. Annealing of the argon matrix up to the highest possible temperature (ca. $35 \mathrm{~K}$ ) did not lead to any changes in the infrared spectrum of $p$-anisaldehyde that could be assigned to conformational changes. This means that within the accessible temperature range the available energy is not enough to overcome either of the internal rotation barriers in $p$-anisaldehyde. As it was previously noticed by Barnes, "there is a rough correlation between the barrier to internal rotation and the lowest temperature at which interconversion was observed". ${ }^{35}$ According to this correlation, "annealing at $35-40 \mathrm{~K}$ would lead to loss of the less stable conformer form molecules with a barrier to rotation of less than $12 \mathrm{~kJ} \mathrm{~mol}^{-1,35,36}$ This puts a lower limit of the barrier (of about $10 \mathrm{~kJ} \mathrm{~mol}^{-1}$ ) that cannot be overcome in an argon matrix during annealing.

On the other hand, xenon matrices can be annealed up to higher temperatures without significant evaporation of the host, aggregation of the sample, or losses in optical properties of the matrix. When the xenon matrix was heated to the temperatures 
TABLE 2: Assignment of the Infrared Spectra of $p$-Anisaldehyde Isolated in Argon and Xenon Matrices ${ }^{a}$

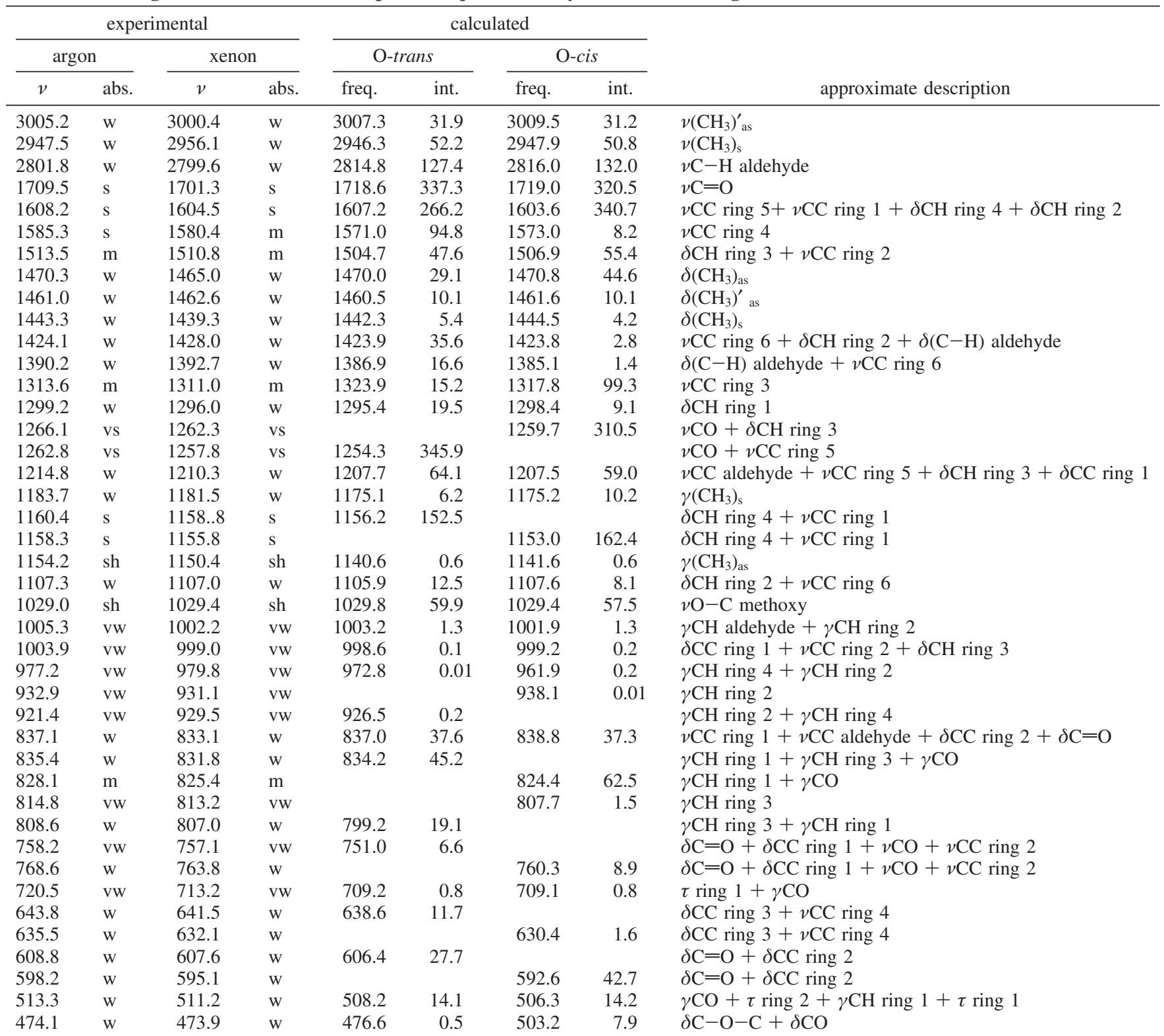

${ }^{a}$ For detailed description of the normal modes in terms of potential energy distribution and complete set of calculated frequencies and infrared intensities, see Supporting Information Tables S1-S3.

of 53-57 K, significant changes occurred in the spectrum of $p$-anisaldehyde. These spectral effects were a clear indication of conversion of the O-cis conformer into the O-trans form. Such temperature-induced changes concerned many doublet bands, and the identification of the conformer of $p$-anisaldehyde being produced and that being consumed was reliably made by comparison of the experimental IR spectra (recorded before and after annealing) with the theoretical spectra calculated for the two conformers of the compound (Figure 4). The observed direction of the conformational conversion (O-cis $\rightarrow$ O-trans) confirms that in the matrices, as in the gas phase, the O-trans form is the most stable conformer of $p$-anisaldehyde.

In view of the relative values of the two barriers for internal rotation in $p$-anisaldehyde, it can be appropriately stated that, in the electronic ground state of this molecule and at cryogenic temperatures, the conformational relaxation during annealing arises from crossing of the lower of the two internal barriers, that is, from the rotation of the methoxy group. Crossing the higher barrier for rotation of the aldehyde group is quite improbable in comparison to crossing of the significantly lower barrier for rotation of the methoxy group.

To prove that the aldehyde group attached to an unsaturated ring does not undergo thermal rotation in matrices, we carried out an additional model experiment on pyrrole-2-carboxaldehyde (P2C). In this experiment, we deposited P2C in a xenon matrix at $30 \mathrm{~K}$. After deposition, the sample of P2C was irradiated with UV-light $(\lambda>234 \mathrm{~nm})$. As it was established previously, ${ }^{37}$ such irradiation converts more than $20 \%$ of the most stable cisconformer into the trans form. These two forms of P2C differ from each other only by the orientation of the aldehyde group and are separated by a barrier of ca. $43 \mathrm{~kJ} \mathrm{~mol}^{-1}$ (see Table 1) and have very distinct spectral signatures. ${ }^{37}$ The xenon matrix containing a mixture of the two P2C conformers was then subjected to annealing, up to $65 \mathrm{~K}$. No conformational change of $\mathrm{P} 2 \mathrm{C}$ could be observed, showing that the thermal rotation of the aldehyde group at cryogenic temperatures is precluded by the high barrier. 


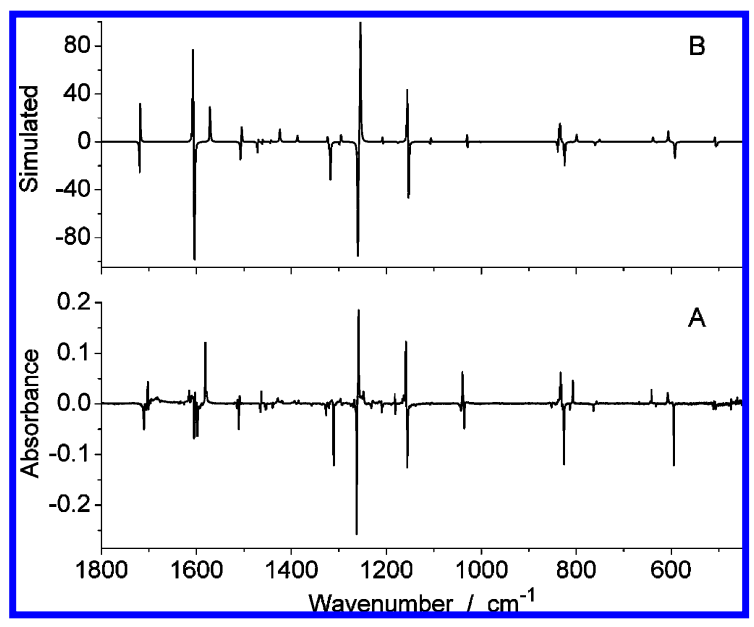

Figure 4. A: Difference IR absorption spectrum showing thermally induced conformational isomerism resulting from annealing of the xenon matrix up to $57 \mathrm{~K}$. Positive bands correspond to the features that increase during annealing, whereas negative bands are decreasing. B: Simulated difference spectrum (O-trans minus O-cis) obtained using Lorentzian functions ( $\mathrm{fwhm}=2 \mathrm{~cm}^{-1}$ ) centered at the calculated (scaled) wavenumbers. The calculated DFT(B3LYP)/6-311++G(d,p) frequencies were scaled by 0.978 .

In a separate experiment, a sample of $p$-anisaldehyde isolated in a xenon matrix was annealed to temperatures higher than 55 $\mathrm{K}$ (to $60 \mathrm{~K}$ and higher temperatures where aggregation starts to occur). The experimental spectra before annealing (at $30 \mathrm{~K}$ ) and at one of the stages of this process (at $57 \mathrm{~K}$ ) are shown in Figure 5, where they are compared to the theoretically predicted spectra of two conformers. The relative spectral position of the bands that are decreasing and increasing during annealing corresponds very well to the predicted spectra of O-cis and O-trans conformers, respectively. In some cases, these bands stay remote by $10 \mathrm{~cm}^{-1}$ or more (see Figure 5). The mutual conversion of these pairs of bands during annealing (and in the opposite direction during the UV-irradiation of the samples, as it will be shown later) evidences the conformational nature of the doublets rather than matrix site-splitting effect.
The conformational ratio remained the same after annealing the sample above $55 \mathrm{~K}$. This result indicates that no further transformation of O-cis to O-trans form could be induced by additional heating. Such situation can take place if a true thermal equilibrium is established between the two conformers, where the minor form has a non-negligible population. It appears interesting to estimate the relative ratio of the two conformers at $57 \mathrm{~K}$ experimentally and deduce their relative stability. The relative amount of the two conformers in the annealed sample was assessed from a series of conformational doublets centered around 1260, 830, and $600 \mathrm{~cm}^{-1}$ (see Figure 5). The integrated areas of the experimental bands due to individual conformers were reduced by the calculated intensities of the corresponding vibrational modes. This permitted to establish that the O-cis/ O-trans conformer ratio upon annealing is about 1:7.2 ( $\pm 20 \%)$, which corresponds to the difference in stabilities of ca. $0.9 \mathrm{~kJ}$ $\mathrm{mol}^{-1}$. This estimate is slightly higher than the theoretically predicted MP2 value of $0.57 \mathrm{~kJ} \mathrm{~mol}^{-1}$ (Table S4). One of the possible explanations is that the O-trans conformer is additionally stabilized in xenon matrices due to its larger dipole moment (the calculated value is $5.3 \mathrm{D}$ ) with respect to the O-cis conformer $(4.3 \mathrm{D})$.

It is interesting to comment on the conformational behavior observed during the cooling after annealing. When the sample was cooled back to $30 \mathrm{~K}$, the conformational ratio did not change, if compared to that obtained at $55 \mathrm{~K}$. The obvious explanation is that the rotamerization barrier in $p$-anisaldehyde is no longer thermally accessible at temperatures lower than 55 $\mathrm{K}$. Hence, annealing and subsequent cooling represent a very efficient tool of control over the conformational populations that can trigger the conformational relaxation.

UV-Induced Conformational Changes. UV-induced mutual conversions of conformers differing in the orientation of their aldehyde group have been observed for several analogues of benzaldehyde. ${ }^{38-40}$ Photochemical rotations were found also for methoxy groups attached to a benzene ring. ${ }^{41}$ It could then be expected that conformational isomerization would also occur for matrix-isolated $p$-anisaldehyde subjected to UV irradiation. Since, for $p$-anisaldehyde isolated in xenon matrices, the

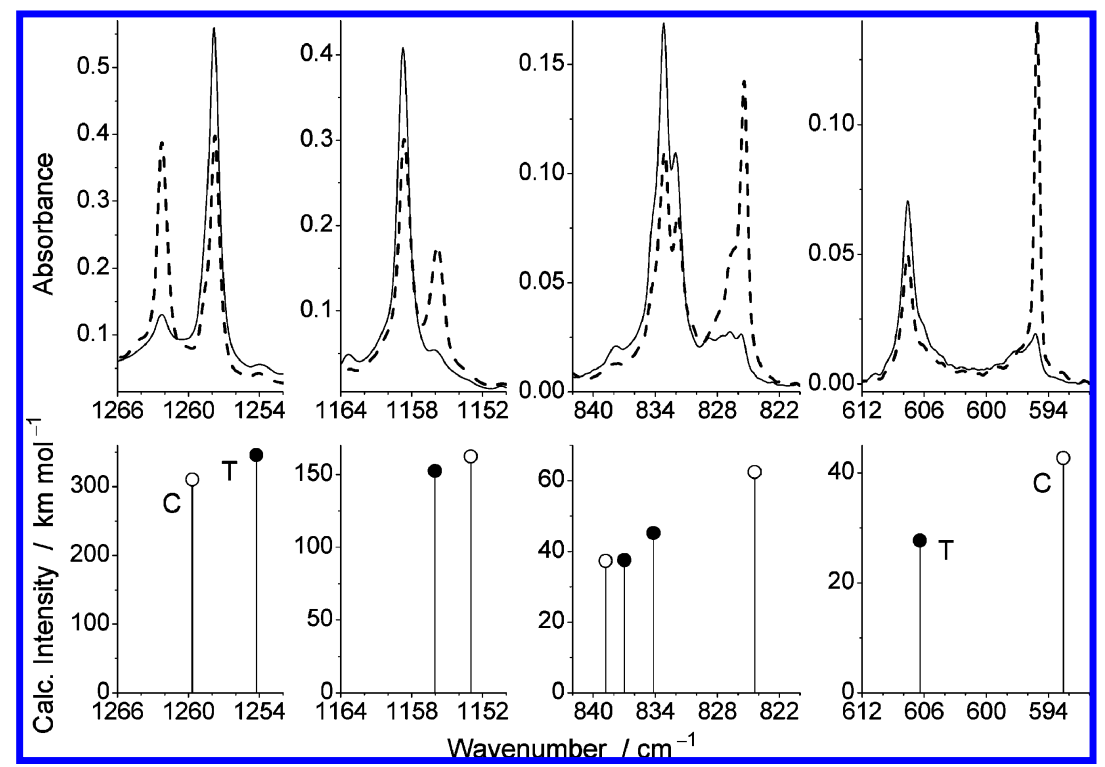

Figure 5. Upper row: Selected regions of the experimental FTIR spectrum of $p$-anisaldehyde isolated in a xenon matrix showing large-scale thermally induced conformational changes occurring upon annealing of the sample from $30 \mathrm{~K}$ (dashed line) to $57 \mathrm{~K}$ (solid line). Lower row: Theoretical spectra of O-trans (full circles, T) and O-cis (open circles, C) conformers calculated at the DFT(B3LYP)/6-311++G(d,p) level. The calculated wavenumbers were scaled by a factor of 0.978 . 


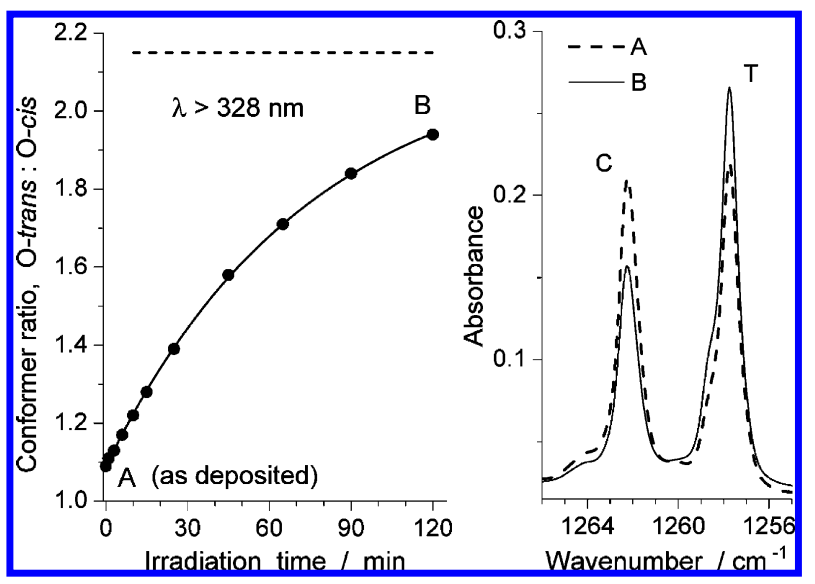

Figure 6. Right: effect of UV-irradiation $(\lambda>328 \mathrm{~nm})$ on the infrared spectra of anisaldehyde isolated in a xenon matrix at $30 \mathrm{~K}$. Dashed trace corresponds to the spectrum of a freshly deposited sample, continuous trace shows the spectrum after two hours of irradiation. " $\mathrm{C}$ " and " $\mathrm{T}$ " designate absorptions of conformers O-cis and O-trans, respectively. Left: Kinetics of the conformational changes. Black circles: ratio of the conformers estimated from the absorptions in the 1264-1256 $\mathrm{cm}^{-1}$ range (shown in the right frame). The integrated experimental band intensities were reduced by the calculated infrared intensities of Cis and Trans conformers. Solid line: exponential fit according to the equation $\left[y=A \exp \left(-x / t_{\mathrm{a}}\right)+y_{0}\right]$, where $A=-1.065 \pm 0.013 ; t_{\mathrm{a}}=$ $74.1 \pm 1.8 ; y_{0}=2.154 \pm 0.014$, and correlation coefficient $R=$ 0.99985. Dashed line: asymptotic limit $\left(y_{0}\right)$.

population ratio of O-cis and O-trans conformers can be also controlled by temperature variation (as described above), we selected the Xe matrix medium for the photochemical studies.

$p$-Anisaldehyde isolated in a xenon matrix was irradiated with continuous-wave, broadband UV light coming from a highpressure $\mathrm{Xe} / \mathrm{Hg}$ lamp fitted with a long-pass $\lambda>328 \mathrm{~nm}$ cutoff filter. As it is revealed by the intensity changes of IR bands characteristic of O-cis and O-trans forms (Figure 6), UV $(\lambda>$ $328 \mathrm{~nm}$ ) irradiation leads to alteration of the O-cis/O-trans population ratio. This ratio changes from the initial value of $\sim 1: 1.1$ (observed after deposition of the matrix) toward a photostationary state with O-cis/O-trans $\approx 1: 2.2$. Hence, with respect to the O-cis/O-trans ratio in a freshly deposited matrix, a net increase of population of the O-trans form at the cost of a decrease of O-cis population was induced by irradiation with $\mathrm{UV}(\lambda>328 \mathrm{~nm})$.

A net transformation in the reverse direction was observed when a Xe matrix with very high O-trans/O-cis ratio (obtained after annealing to $57 \mathrm{~K}$ and subsequent cooling to $30 \mathrm{~K}$ ) was subjected to the same UV $(\lambda>328 \mathrm{~nm})$ light (see Figure 7). Under such conditions, a net transformation of O-trans isomer into O-cis form occurred. This clearly demonstrates that irradiation of matrix isolated $p$-anisaldehyde with $\mathrm{UV}(\lambda>328$ $\mathrm{nm}$ ) light leads to the same photostationary state, independent of the initial population ratio of the two conformers. Direct observations of UV induced O-cis $\rightarrow \mathrm{O}$-trans as well as O-trans $\rightarrow$ O-cis net transformations provide an undisputable proof of photoreversibility of the O-cis $\leftrightarrow$ O-trans conversions. In a separate experiment, UV irradiation of the annealed matrix without subsequent cooling, that is, at $57 \mathrm{~K}$, was attempted. It did not induce any conformational changes in the samples and the O-cis/O-trans conformer ratio remained about 1:7, that is, the thermal processes dominated under these conditions.

$p$-Anisaldehyde isolated in a Xe matrix at $30 \mathrm{~K}$ was also irradiated with broadband UV light of shorter $(\lambda>295 \mathrm{~nm})$ wavelengths. Irradiation at such wavelength range leads (see Figure 8 ) to a final population ratio O-cis/O-trans $\approx 1: 1.4$. This

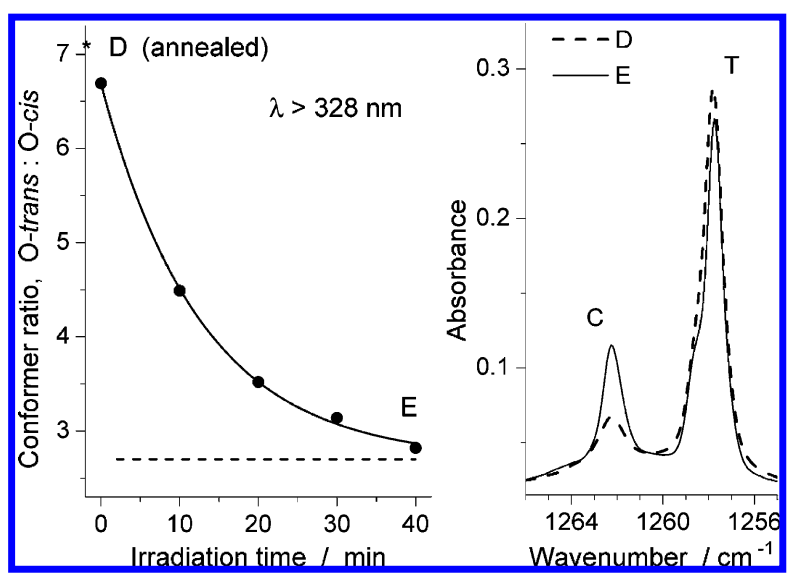

Figure 7. Right: effect of UV-irradiation $(\lambda>328 \mathrm{~nm})$ on the infrared spectra of anisaldehyde isolated in a xenon matrix. Dashed trace corresponds to the spectrum of a sample annealed to $57 \mathrm{~K}$ and subsequently cooled to $30 \mathrm{~K}$, continuous trace shows the spectrum after forty minutes of irradiation. "C" and "T" designate absorptions of conformers O-cis and O-trans, respectively. Left: Kinetics of the conformational changes. Black circles: ratio of the conformers estimated from the absorptions in the $1264-1256 \mathrm{~cm}^{-1}$ range (shown in the right frame). The integrated experimental band intensities were reduced by the calculated infrared intensities of Cis and Trans conformers. Solid line: exponential fit according to the equation $\left[y=D \exp \left(-x / t_{\mathrm{d}}\right)+\right.$ $y_{2}$ ], where $D=3.985 \pm 0.090 ; t_{\mathrm{d}}=12.68 \pm 0.77 ; y_{2}=2.699 \pm 0.077$, and correlation coefficient $R=0.99925$. Dashed line: asymptotic limit $\left(y_{2}\right)$.

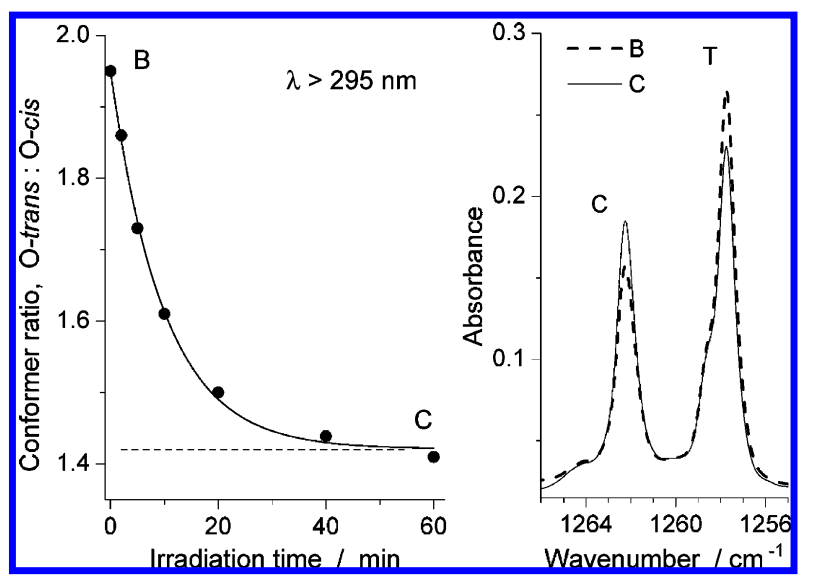

Figure 8. Right: Effect of UV-irradiation $(\lambda>295 \mathrm{~nm})$ on the infrared spectra of anisaldehyde isolated in a xenon matrix at $30 \mathrm{~K}$. Dashed trace (B) is the same as trace B in Figure 6, continuous trace shows the spectrum after one hour of irradiation. " $\mathrm{C}$ " and " $\mathrm{T}$ " designate absorptions of conformers O-cis and O-trans, respectively. Left: Kinetics of the conformational changes. Black circles: ratio of the conformers estimated from the absorptions in the $1264-1256 \mathrm{~cm}^{-1}$ range (shown in the right frame). The integrated experimental band intensities were reduced by the calculated infrared intensities of Cis and Trans conformers. Solid line: exponential fit according to the equation $\left[y=B \exp \left(-x / t_{\mathrm{b}}\right)+y_{1}\right]$, where $B=0.529 \pm 0.011 ; t_{\mathrm{b}}=9.87$ $\pm 0.56 ; y_{1}=1.421 \pm 0.008$, and correlation coefficient $R=0.99825$. Dashed line: asymptotic limit $\left(y_{1}\right)$.

photostationary ratio is not the same as that achieved after UV $(\lambda>328 \mathrm{~nm})$ excitation. In this comparison, the photostationary relative population of O-cis form (recorded after prolonged $\lambda>$ $295 \mathrm{~nm}$ irradiation) is significantly higher. The dependence of the photostationary ratio of O-cis and O-trans conformers on the wavelength of applied UV light was further illustrated by the effects of irradiation of matrix-isolated $p$-anisaldehyde monomers with $\mathrm{UV}(\lambda>234 \mathrm{~nm})$ light. At the photostationary 


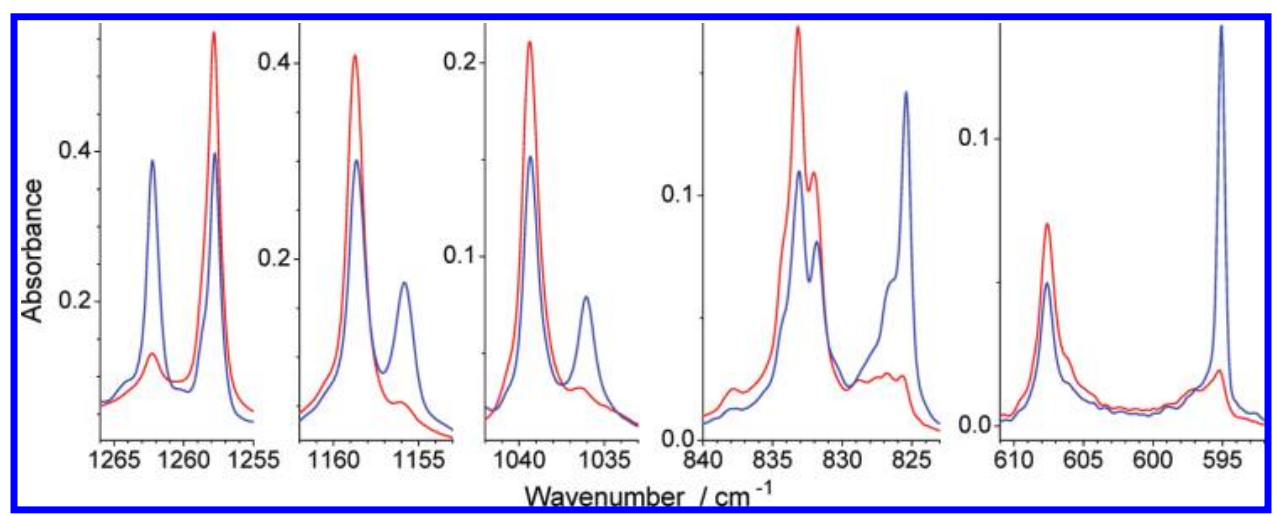

Figure 9. Blue trace: fragments of the IR spectrum recorded after UV $(\lambda>234 \mathrm{~nm})$ irradiation of $p$-anisaldehyde isolated in a Xe matrix at $30 \mathrm{~K}$ (estimated population ratio O-cis/O-trans $\cong 1: 0.89$ ). Red trace: corresponding fragments of the spectrum recorded (in a separate experiment) after annealing of a Xe matrix to $57 \mathrm{~K}$ (estimated population ratio $\mathrm{O}$-cis/O-trans $\cong 1: 7.2$ )

TABLE 3: Energy of Vertical Absorption $(\Delta E)$ and Oscillator Strength $(f)$ Calculated using the TD-DFT(B3LYP)/6-311 ++G(d,p) Method at the Ground State Equilibrium Geometries ${ }^{a}$ of O-trans and O-cis forms of p-Anisaldehyde

\begin{tabular}{ccccccc}
\hline & & \multicolumn{2}{c}{ O-trans } & & \multicolumn{2}{c}{ O-cis } \\
state & type & $\Delta E(\mathrm{eV})$ & $f$ & & $\Delta E(\mathrm{eV})$ & $f$ \\
\hline $\mathrm{S}_{0}\left(\mathrm{~A}^{\prime}\right)$ & & 0.0 & & & 0.002 & \\
$\mathrm{~S}_{1}\left(\mathrm{~A}^{\prime \prime}\right)$ & $\mathrm{n} \pi^{*}$ & 3.77 & 0.0001 & & 3.76 & 0.0001 \\
$\mathrm{~S}_{2}\left(\mathrm{~A}^{\prime}\right)$ & $\pi \pi^{*}$ & 4.69 & 0.338 & & 4.63 & 0.350 \\
$\mathrm{~S}_{3}\left(\mathrm{~A}^{\prime}\right)$ & $\pi \pi^{*}$ & 4.79 & 0.065 & & 4.77 & 0.007
\end{tabular}

${ }^{a}$ The ground-state equilibrium geometries of O-trans and O-cis forms of $p$-anisaldehyde were optimized at the DFT(B3LYP)/ 6-311++G(d,p) level.

state induced by UV $(\lambda>234 \mathrm{~nm})$ irradiation, the population of O-cis form is higher than that of O-trans conformer (see Figure 9).

The energies of vertical transitions from $S_{0}$ to the lowestenergy excited singlet states $S_{1}, S_{2}$, and $S_{3}$ were calculated at the TD-DFT(B3LYP) level for the O-trans and O-cis forms of $p$-anisaldehyde. The results of these calculations (presented in Table 3 ) show that among the excitations to the $S_{1}, S_{2}$, and $S_{3}$ states only the transition to $S_{2}$ has substantial oscillator strength. Direct $\mathrm{S}_{0} \rightarrow \mathrm{S}_{1}$ excitation has $n \pi^{*}$ character, and although it is not strictly forbidden, it should be very ineffective due to the very low value of the oscillator strength. Hence, in order to effectively excite $p$-anisaldehyde, higher energy UV light allowing $S_{0} \rightarrow S_{2}\left(\pi \pi^{*}\right)$ transition should be applied.

The profiles of potential energies of $S_{0}, S_{1}, S_{2}$ and $S_{3}$ states were calculated as a function of aldehyde group rotation (represented by $\mathrm{C}-\mathrm{C}-\mathrm{C}=\mathrm{O}$ dihedral angle) and as a function of methoxy group rotation (represented by $\mathrm{C}-\mathrm{C}-\mathrm{OC}$ dihedral angle). These profiles (computed at the TDDFT level) are presented in Figure 10. Along the whole $180^{\circ}$ turn of the aldehyde and methoxy groups, the excited $S_{1}, S_{2}$, and $S_{3}$ states are well separated (by more than $3.5 \mathrm{eV}$ ) from the ground $S_{0}$ state. Therefore, no conical intersections between these excited states and the electronic ground state are expected in the probed regions of the potential energy surfaces. The UV-induced O-trans $\leftrightarrow$ O-cis isomerizations can be explained by the very flat potential energy surfaces in $S_{2}$ (for the aldehyde group rotation, barrier of only $0.21 \mathrm{eV}$ ) and in $S_{1}$ and $S_{3}$ (for the methoxy group rotation, barriers of only 0.13 and $0.15 \mathrm{eV}$, respectively). To cross such low barriers, very little excess of excitation energy is necessary. Moreover, the barriers in $S_{0}$ are very small in comparison with the energy of electronic excitation

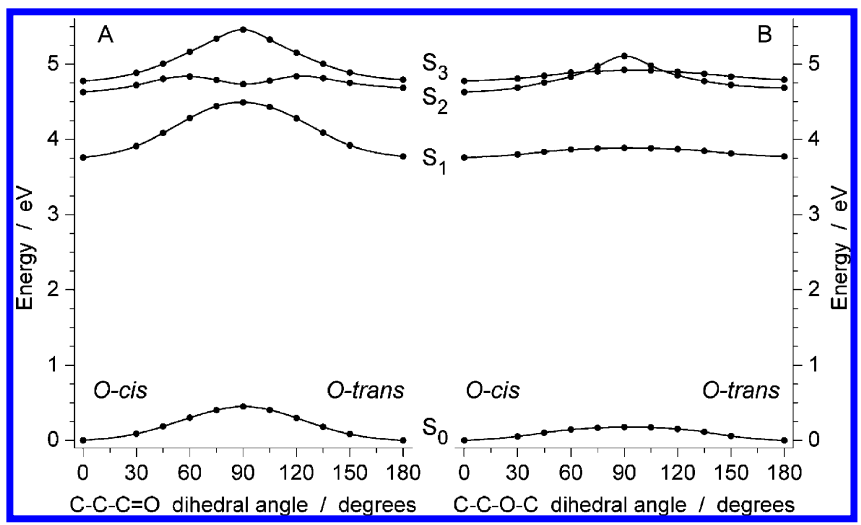

Figure 10. TD-DFT profiles of the lowest-energy singlet states of $p$-anisaldehyde calculated at the (B3LYP)/6-311++G(d,p) level as functions of internal rotation of the aldehyde group (determined by the $\mathrm{C}-\mathrm{C}-\mathrm{C}=\mathrm{O}$ dihedral angle, frame $\mathrm{A}$ ) and of internal rotation of the methoxy group (determined by the $\mathrm{C}-\mathrm{C}-\mathrm{O}-\mathrm{C}$ dihedral angle, frame B).

to $S_{1}$ or $S_{2}$. Hence, these barriers can also be easily crossed during radiationless dissipation of the excitation energy. The profiles presented in Figure 10 can explain the UV-induced $\mathrm{O}$-trans $\leftrightarrow \mathrm{O}$-cis isomerizations observed for $p$-anisaldehyde and (by analogy) the photochemical trans $\leftrightarrow$ cis isomerizations previously observed for compounds with the aldehyde or with the methoxy groups attached to aromatic rings. ${ }^{38-41}$

The presented observations indicate that the ratio of conformers at a photostationary state is specific to every wavelength of UV light used for irradiation. The observed net direction of the photoinduced conversions is dependent on the wavelength range of the applied UV light as well as on the initial population ratio of the conformers. Hence, the relative amount of the two $p$-anisaldehyde conformers in cryogenic matrix can be efficiently varied in both directions by irradiating the sample with UV light at different wavelength ranges or by combined use of UV irradiation and thermal annealing of the matrix. The relative dominance of the O-trans form in the photostationary states produced with excitations at longer wavelengths (see Figures 6 and 7) can be explained by a higher consumption of the O-cis form, since it has lower excitation energy to the $S_{2}$ state (see Table 3). On the contrary, the prevalence of the O-cis form in the photostationary equilibria produced with shorter excitation wavelengths (see Figure 9) can be explained by a higher oscillator strength of the O-trans form related with the transition to the $\mathrm{S}_{3}$ state.

It is important to stress that in all experiments described above, except the UV light with $\lambda>234 \mathrm{~nm}$, no processes other 


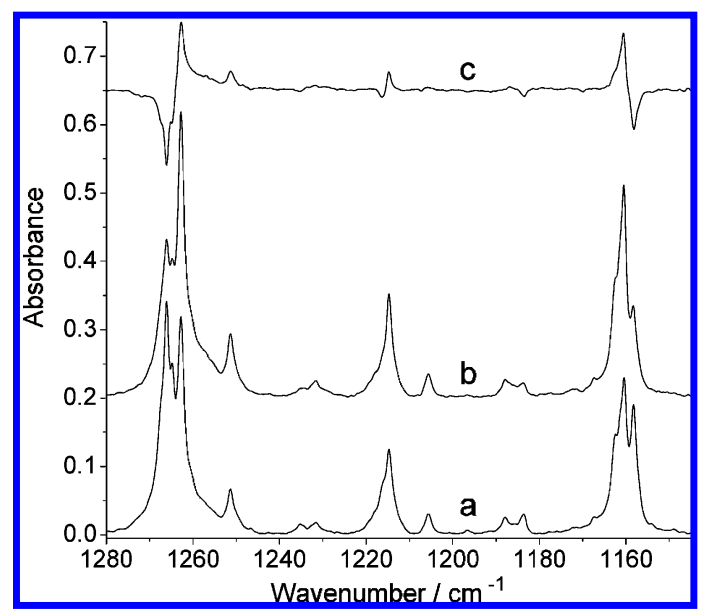

Figure 11. (a) $1280-1145 \mathrm{~cm}^{-1}$ region of the spectrum of $p$-anisaldehyde isolated in an Ar matrix at $10 \mathrm{~K}$; (b) spectrum after 30 min irradiation by a narrowband laser light of $\lambda=280 \mathrm{~nm}$; (c) difference spectrum: $\mathrm{c}=\mathrm{b}-\mathrm{a}$.

than conformational isomerization occurred in a significant way. In particular, no bands due to other photoproducts were observed in the spectra of the irradiated Xe matrices (or they are of very small intensity), and no indications of significant aggregation were found in the spectra of the annealed matrices. Regarding the irradiation with UV $(\lambda>234 \mathrm{~nm})$ light, the conformational transformation was concomitant with the decomposition of the compound. However, the former process arrived to the photostationary state very fast, and the conformational ratio did not change during the photodecomposition, despite the absolute amounts of both conformers had decreased. This situation is similar to what was observed for matrix-isolated pyrrole-2carbaldehyde (P2C). ${ }^{37}$

Irradiation experiments were also attempted for the compound isolated in an argon matrix. By analogy with $\mathrm{P} 2 \mathrm{C},{ }^{37}$ it could be expected that the compound isolated in solid argon should be converted to other species slower than in xenon. Nevertheless, only very short irradiation with narrowband UV light afforded the conformational isomerization without significant loss of the $p$-anisaldehyde reactant isolated in an argon matrix. However, even with this more sophisticated approach, the UV control of conformational distribution in argon matrix proved to be limited. Indeed, in Ar matrix the isomerization could be induced only in one direction: irradiation at $280 \mathrm{~nm}$ was found (see Figure 11) to convert the O-cis conformer into the O-trans form (for a matrix irradiated directly after deposition, hence with relatively large initial amount of the O-cis form). This result can be interpreted assuming that irradiation at $280 \mathrm{~nm}$ shifts the ratio of conformers isolated in an Ar matrix toward the photostationary state characteristic of this wavelength. It would be interesting to test the result of irradiating a sample with an excess of O-trans form relative to the position of the photostationary equilibrium, which should lead to the reverse process. However, in an Ar matrix there is no way to attain such situation, since annealing is not usable to this end.

The narrowband irradiation at short wavelengths to attempt to selectively convert the O-trans form into the O-cis is impracticable: irradiation of $p$-anisaldehyde in an Ar matrix at wavelengths shorter than $280 \mathrm{~nm}$ led to extensive production of new chemical species absorbing in the vicinity of $2140 \mathrm{~cm}^{-1}$, which can be attributed to ketene group $(\mathrm{C}=\mathrm{C}=\mathrm{O})$ or carbon monoxide (or both).

\section{Conclusions}

Two conformers of $p$-anisaldehyde, O-trans and O-cis, were trapped from the room temperature gas phase in low-temperature argon and xenon matrices. Both conformers were found to be almost equally populated in the matrices as it could be expected, assuming that the gas phase conformational distribution could be efficiently trapped in solidifying argon or xenon. Upon annealing of a xenon matrix to $57 \mathrm{~K}$, molecules of $p$ anisaldehyde were found to undergo a facile $\mathrm{O}$-cis $\rightarrow$ O-trans conversion until the conformational populations reached a true thermal equilibrium. UV irradiation of a xenon matrix induced mutual transformations of $p$-anisaldehyde conformers. The photostationary states were obtained, with the final ratio of conformers specific to the wavelength of the applied UV light. The net direction of the observed O-trans $\rightarrow$ O-cis or O-cis $\rightarrow$ O-trans photoinduced population changes was dependent on the range of UV radiation used and on the initial population ratio of the two conformers.

The obtained results also indicate that the photochemistry of $p$-anisaldehyde depends on the matrix medium. For the compound isolated in solid argon, we were only able to induce (using a narrowband laser source tuned at $\lambda=280 \mathrm{~nm}$ ) conformational isomerization in one $\mathrm{O}$-cis $\rightarrow \mathrm{O}$-trans direction. The reverse process could be expected to be induced by irradiation at shorter wavelengths, but in argon matrix irradiation at these wavelengths led to extensive production of new chemical species, so that this method is not usable as a way to induce the reverse O-trans $\rightarrow \mathrm{O}$-cis process.

The bands observed in the infrared spectra of matrix-isolated $p$-anisaldehyde were assigned to the O-trans or O-cis conformers on the basis of intensity changes occurring upon annealing and/ or UV irradiation of the sample. The interpretation of the spectra was aided by the results of the analysis of theoretically predicted normal modes.

Acknowledgment. This investigation has been partially supported by the Portuguese Science Foundation (FCT: Project PTDC/QUI/71293/2006 and Grant SFRH/BPD/31840/2006).

Supporting Information Available: Figure S1, with the adopted atom labeling scheme for O-cis and O-trans conformers of $p$-anisaldehyde. Table S1, providing definitions of the internal coordinates used in the normal-mode analyses. Tables S2 and $\mathrm{S} 3$, providing the DFT(B3LYP)/6-311++G(d,p) calculated theoretical wavenumbers, infrared intensities, and potential energy distributions resulting from the normal coordinate analysis calculations. Table S4 (extended version of Table 1), providing absolute and relative energies of the stationary points calculated for anisaldehyde and model compounds at the DFT(B3LYP) and MP2 levels of theory using the $6-311++G(d, p)$ basis set. This material is available free of charge via Internet at http://pubs.acs.org.

\section{References and Notes}

(1) Narukulla, R.; Shuker, D. E. G.; Xu, Y. Z. Nucleic Acids Res. 2005, $33,1767-1778$.

(2) Baricevic, D.; Sosa, S.; DellaLoggia, R.; Tubaro, A.; Simonovska, B.; Krasna, A.; Zupancic, A. J. Ethnopharmacol. 2001, 75, 125-132.

(3) Liberko, C. A.; Shearer, J. J. Chem. Educ. 2000, 77, 1204-1205.

(4) Shenoy, D. K. Am. J. Phys. 1994, 62, 858-859.

(5) Kitajima, N.; Sunaga, S.; Moro-Oka, Y.; Yoshikuni, T.; Akada, M.; Tomotaki, Y.; Taniguchi, M. Bull. Chem. Soc. Jpn. 1988, 61, 967971.

(6) Ribeiro-Claro, P. J. A.; Batista de Carvalho, L. A. E.; Amado, A. M. J. Raman Spectrosc. 1997, 28, 867-872.

(7) Karger, N.; da Costa, A. M. A.; Ribeiro-Claro, P. J. A. J.Phvs. Chem. A 1999, 103, 8672-8677. 
(8) Puranik, P. G. J. Chem. Phvs. 1957, 26, 601-603.

(9) Melandri, S.; Maris, A.; Favero, P. G.; Favero, L. B.; Caminati, W.; Meyer, R. J. Mol. Spectrosc. 1997, 185, 374-383.

(10) Bohn, R. K.; Farag, M. S.; Ott, C. M.; Radhakrishnan, J.; Sorenson, S. A.; True, N. S. J. Mol. Struct. 1992, 268, 107-121.

(11) Brunvoll, J.; Bohn, R. K.; Hargittai, I. J. Mol. Struct. 1985, 129 $81-91$.

(12) Sharma, A.; Reva, I.; Fausto, R. J. Am. Chem. Soc. 2009, 131, 8752-8753.

(13) Jarmelo, S.; Fausto, R. J. Mol. Struct. 2006, 786, 175-181.

(14) Fausto, R.; Khriachtchev, L.; Hamm, P. Conformational Changes in Cryogenic Matrices. In Physics and Chemistry at Low Temperatures; Khriachtchev, L., Ed.; Pan Stanford Publishing: London, 2010; ISBN: 978981-4267-51-9.

(15) Maçôas, E. M. S.; Khriachtchev, L.; Fausto, R.; Räsänen, M. $\underline{J}$ Phvs. Chem. A 2004, 108, 3380-3389.

(16) Becke, A. D. Phys. Rev. A 1988, 38, 3098-3100.

(17) Lee, C. T.; Yang, W. T.; Parr, R. G. Phvs. Rev. B 1988, 37, 785789.

(18) Vosko, S. H.; Wilk, L.; Nusair, M. Can. J. Phys. 1980, 58, 1200 1211

(19) Frisch, M. J.; Trucks, G. W.; Schlegel, H. B.; Scuseria, G. E.; Robb, M. A.; Cheeseman, J. R.; Montgomery, J. A., Jr.; Vreven, T.; Kudin, K. N.; Burant, J. C.; Millam, J. M.; Iyengar, S. S.; Tomasi, J.; Barone, V.; Mennucci, B.; Cossi, M.; Scalmani, G.; Rega, N.; Petersson, G. A.; Nakatsuji, H.; Hada, M.; Ehara, M.; Toyota, K.; Fukuda, R.; Hasegawa, J.; Ishida, M.; Nakajima, T.; Honda, Y.; Kitao, O.; Nakai, H.; Klene, M.; Li, X.; Knox, J. E.; Hratchian, H. P.; Cross, J. B.; Bakken, V.; Adamo, C.; Jaramillo, J.; Gomperts, R.; Stratmann, R. E.; Yazyev, O.; Austin, A. J.; Cammi, R.; Pomelli, C.; Ochterski, J. W.; Ayala, P. Y.; Morokuma, K.; Voth, G. A.; Salvador, P.; Dannenberg, J. J.; Zakrzewski, V. G.; Dapprich, S.; Daniels, A. D.; Strain, M. C.; Farkas, O.; Malick, D. K.; Rabuck, A. D. Raghavachari, K.; Foresman, J. B.; Ortiz, J. V.; Cui, Q.; Baboul, A. G.; Clifford, S.; Cioslowski, J.; Stefanov, B. B.; Liu, G.; Liashenko, A.; Piskorz, P.; Komaromi, I.; Martin, R. L.; Fox, D. J.; Keith, T.; Al-Laham, M. A.; Peng, C. Y.; Nanayakkara, A.; Challacombe, M.; Gill, P. M. W.; Johnson, B.; Chen, W.; Wong, M. W.; Gonzalez, C.; Pople, J. A. Gaussian 03, revision C.02; Gaussian, Inc.: Wallingford, CT, 2004.

(20) Møller, C.; Plesset, M. S. Phvs. Rev. 1934, 46, 618-622. 464.

(21) Bauernschmitt, R.; Ahlrichs, R. Chem. Phvs. Lett. 1996, 256, 454-
(22) Stratmann, R. E.; Scuseria, G. E.; Frisch, M. J. J. Chem. Phvs. 1998, 109, 8218-8224.

(23) Schachtschneider, J. H.; Mortimer, F. S., Vibrational Analysis of Polyatomic Molecules. VI. FORTRAN IV Programs for Solving the Vibrational Secular Equation and for the Least-Squares Refinement of Force Constants. Project No. 31450. Structural Interpretation of Spectra, Technical Report No. 57-65; Shell Development Co.: Emeryville, CA, 1969.

(24) Pulay, P.; Fogarasi, G.; Pang, F.; Boggs, J. E. J. Am. Chem. Soc.

1979, 101, 2550-2560.

(25) Hiremath, C. S.; Yenagi, J.; Tonannavar, J. Spectrochim. Acta, A 2007, 68, 710-717.

(26) Meier, R. J.; Koglin, E. Chem. Phvs. Lett. 2002, 353, 239-243.

(27) Sancho-García, J. C. J. Chem. Phys. 2006, 124, 124112.

(28) Lister, D. G. J. Mol. Struct. 1980, 68, 33-40.

(29) Spellmeyer, D. C.; Grootenhuis, P. D. J.; Miller, M. D.; Kuyper,

L. F.; Kollman, P. A. J. Phvs. Chem. 1990, 94, 4483-4491.

(30) Vincent, M. A.; Hillier, I. H. Chem. Phvs. 1990, 140, 35-40.

(31) Speakman, L. D.; Papas, B. N.; Woodcock, H. L.; Schaefer, H. F., III J. Chem. Phvs. 2004, 120, 4247-4250.

(32) Reva, I. D.; Stepanian, S. G.; Adamowicz, L.; Fausto, R. Chem. Phvs. Lett. 2003, 374, 631-638.

(33) Reva, I.; Simão, A.; Fausto, R. Chem. Phvs. Lett. 2005, 406, 126136.

(34) Breda, S.; Reva, I.; Lapinski, L.; Fausto, R. J. Phys. Chem. A 2006, 110, 11034-11045.

(35) Barnes, A. J. J. Mol. Struct. 1984, 113, 161-174.

(36) It must be noted, however, that this correlation should be taken very carefully, since it was deduced for experiments conducted mainly in argon and nitrogen matrices, and also for rotation of the intramolecular fragments other than methoxy or aldehyde group. Our previous study on cyanoacetic acid and methylcyanoacetate (ref 32) showed that every particular case of annealing should be analyzed individually.

(37) Giuliano, B. M.; Reva, I.; Fausto, R. J.Phvs. Chem. A 2010, 114, 2506-2517.

(38) Ohno, K.; Itoh, T. J. Phvs. Chem. A 2007, 111, 7048-7056.

(39) Ohno, K.; Itoh, T.; Yokota, C.; Katsumoto, Y. J. Mol. Struct. 2006, $825,143-150$.

(40) Itoh, T.; Akai, N.; Ohno, K. J. Mol. Struct. 2006, 786, 39-45.

(41) Akai, N.; Kudoh, S.; Nakata, M. J. Phvs. Chem. A 2003, 107, $2635-2641$.

JP102129S 